\title{
Expatriados empresariales y comunidades extranjeras en Monterrey: los casos español y japonés
} Corporate expatriates and foreign communities in Monterrey:
The Spanish and Japanese cases Centro de Estudios Interculturales del Noreste Universidad Regiomontana, Monterrey, México http://orcid.org/o000-0002-0742-3757

Juan Antonio Doncel de la Colina** Centro de Estudios Interculturales del Noreste Universidad Regiomontana, Monterrey, México http://orcid.org/0000-0002-7779-027x

ISSN: ISSN-OI85-4259; e- ISSN: 2007-9176 DOI: http://dx.doi.org/10.28928/revistaiztapalapa/852018/aotı/sordomolinajc/donceldelacolinaja

\begin{abstract}
Resumen
A través de una metodología cualitativa, basada en entrevistas en profundidad y observaciones participantes, presentamos un análisis comparativo de cómo las condiciones laborales y el respaldo que brindan las empresas trasnacionales japonesas y españolas que transfieren personal de sus países de origen repercuten en la cohesión y las dinámicas comunitarias de los connacionales radicados en un centro urbano como Monterrey. Después de contextualizar social, empírica y teóricamente nuestro problema de investigación, así como de detallar nuestro enfoque metodológico, presentamos los resultados obtenidos en cuatro apartados. Los resultados mostraron que las modalidades e intensidades del respaldo empresarial condicionan de manera muy contrastante, en función de la nacionalidad de los expatriados de que se trate, las dinámicas de las comunidades de migrantes ligados a las empresas trasnacionales.

Palabras clave: migración calificada, espacios trasnacionales, cultura empresarial, cohesión grupal, administración global
\end{abstract}

\begin{abstract}
Through a qualitative approach, based on in-depth interviews and participant observations, we present a comparative analysis of how the occupational conditions and the support provided by Japanese and Spanish transnational corporations that transfer personnel from their countries of origin, influences the cohesion and community dynamics of nationals living in an urban center such as Monterrey. First, we contextualize, socially, empirically and theoretically, our research problem. Then, a detailed methodological description is offered. Finally, we present our results in four sections. These results indicated that modalities and intensities of the company support, depending on the nationality of the expatriates in question, shape up highly contrasting migrant communities dynamics.
\end{abstract}

Keywords: Skilled migration, transnational spaces, organizational culture, group cohesion, global Management

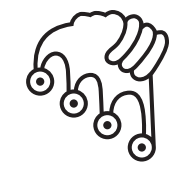

\section{IZTAPALAPA}

Agua sobre lajas

**jdoncel@u-erre.mx 


\section{Introducción}

ntre 2010 y 2or4, desde el Centro de Estudios Interculturales del Noreste,
realizamos una investigación sobre la cohesión grupal y la relación con la
sociedad receptora de in comunidades extranjeras que residen en el área metropolitana de Monterrey (AMM). ${ }^{1}$ Encontramos que una parte importante de la migración de ciertas nacionalidades estaba conformada por técnicos y administradores de alto nivel que trabajan para empresas con matrices en sus países de origen, situación condicionada por el perfil empresarial e industrial de la ciudad. Otro hallazgo fue que la concentración en torno a ámbitos ocupacionales y centros de trabajo representa uno de los factores que influyen para la cohesión grupal de los connacionales migrantes (Doncel, 2013). Sin embargo, se reconoció la necesidad de analizar más detalladamente las condiciones laborales específicas de algunos sectores de cada migración y su impacto en las dinámicas comunitarias globales. Para ello, en este nuevo trabajo decidimos profundizar en la información previamente generada.

Aunque el estudio de la migración de personas con alta calificación profesional data de varias décadas, se han privilegiado los abordajes de sus aspectos económicos, ya que se le considera un fenómeno socialmente menos problemático que otros flujos migratorios (Peixoto, 200I). En la investigación sobre los expatriados empresariales se intensifican estos presupuestos, al parecer un perfil menos conflictivo por tratarse de estancias temporales gestionadas corporativamente. En consecuencia, estos flujos han sido estudiados preferentemente "dentro de una lógica de restructuración global de los procesos productivos y de la división internacional del trabajo" (Mendoza et al., 2016: 3) y como una career-path migration (Salt, 1983).

Paulatinamente se ha superado esa visión economicista y se ha reconocido la migración calificada como un fenómeno de movilidad humana complejo y cada vez

1 Proyecto auspiciado a través de la convocatoria de Investigación Básica SEP-CONACYT 2009, titulado "Dinámicas de interacción, integración y conflicto de las comunidades de extranjeros en Monterrey". 
más relevante. Entre las nuevas temáticas para su estudio se incorporan las dimensiones social y afectiva (Mendoza et al., 2016), predominantemente su integración a las sociedades receptoras (Bermúdez, 20Io), y, en el caso específico de los expatriados, su competencia intercultural (Black y Mendenhall, 1990; Mead, 2009). Esto expresa un interés por su bienestar personal pero, claramente, también la preocupación por evitar los altos costos que supone un retorno anticipado, tema constante en las encuestas sobre expatriación realizadas por agencias privadas. ${ }^{2}$

Sin embargo, la adaptación de un expatriado al contexto social al que es asignado, debido a la brevedad de su estancia, no depende solamente de su ajuste a la sociedad receptora y a sus pautas culturales, sino también del establecimiento de dinámicas grupales con otros connacionales migrantes que le proporcionen un espacio de sociabilidad más familiar, orientación y apoyo (Williams, 2004). Ahora bien, mientras algunas compañías atienden la formación intercultural de su personal asignado al extranjero, hay escasa evidencia de que se preocupen por su integración con otros connacionales. ${ }^{3}$ Por lo tanto, nos hemos interesado en explorar cómo la condición de expatriados empresariales de un sector significativo de dos migraciones en el Амм determina las dinámicas sociales internas de toda la comunidad de dichas nacionalidades. Nuestro objetivo es analizar cómo las condiciones de expatriación y el respaldo que brindan las empresas que transfieren personal de sus países de origen repercuten en la cohesión y las dinámicas comunitarias de los connacionales radicados en un centro urbano como Monterrey.

Decidimos concentrarnos en el caso de las migraciones japonesa y española pues, además de la relevancia cuantitativa y simbólica de ese sector de expatriados, en trabajos previos ya comparamos el grado de cohesión de estas comunidades (Doncel, 20I3) y analizamos cómo la distancia cultural condiciona su integración a la sociedad receptora (Doncel, 2015b). Si bien inicialmente hallamos la correlación esperada entre grado de cohesión grupal y respuesta adaptativa a la sociedad de acogida en casos como el español o el japonés (una respuesta más integradora con la sociedad de acogida y menos cohesionada intragrupalmente en el primer caso y más segre-

2 Por ejemplo, la encuesta de 2016 de Brookfield Global Relocation Services (http://globalmobilitytrends.bgrs.com/) señala la adaptación del expatriado y su familia como uno de los tres elementos más desafiantes para la administración exitosa de la movilidad internacional del personal.

3 Aunque los datos no son generalizables, en una encuesta a expatriados españoles (en el exterior) y extranjeros (en España) se encontró que consideran que la empresa se preocupa más por su desarrollo y adaptación personal que por ponerle en contacto con otros expatriados (Álvarez y Gómez, 2008). 
gada en el segundo), otros resultados no tan concluyentes con otras comunidades aconsejaron introducir la variable "distancia cultural" entre la sociedad de origen y la receptora. Así, seleccionamos los casos japonés y español de las ir comunidades iniciales por considerarlos paradigmáticos en lo que se refiere a cercanía y lejanía cultural respecto de la sociedad mexicana. Esta variable se consideró tanto desde las diferencias reales como desde las percibidas, destacando como las más evidentes entre las diferencias reales las relativas al idioma y a las creencias religiosas.

Por una parte, el migrante español enfrenta un proceso de adaptación mucho más asequible que el japonés, cuyo idioma materno no es ni remotamente parecido al español. Además, entre México y España se da una continuidad histórica mediada por su tradición católica romana (a pesar del fortalecimiento en México de otras denominaciones cristianas y del avance de la secularización de la sociedad española, más cercana a la marcada secularización detectada entre los migrantes japoneses). Por otra parte, en el caso de Japón es evidente la lejanía en el terreno religioso. Tal y como escribimos en su momento:

El hecho de contraponer una religión como la católica, tan orientada al colectivismo y a la práctica pública, frente al sincretismo religioso japonés (mezcla de sintoísmo, budismo y culto a los ancestros), religión de vivencia mucho más subjetiva, individual y de íntima celebración, también tiene su reflejo en los valores soterrados, en la ética dominante, en la forma de regular las cotidianas interacciones sociales, etc. En este sentido, la comunicación intercultural, más allá de la mediada por el lenguaje, entre japoneses y mexicanos viene dificultada por una concepción jerárquica y del respeto en las relaciones sociales muy estricta en el caso japonés, así como por una comunicación no verbal también muy diferenciada entre mexicanos y japoneses (por ejemplo, en lo que atañe al contacto físico, mucho más restringido en el caso japonés) (Doncel, 2015b: 186).

En nuestro trabajo previo resaltamos particularmente la percepción de dicha distancia y las situaciones de conflicto intercultural entre los migrantes japoneses y españoles afincados en Monterrey, siendo mucho más recurrentes las situaciones de conflicto narradas por informantes japoneses. Ahora bien, para el éxito o fracaso de la comunicación intercultural encontramos tan determinante la distancia cultural como las circunstancias específicas de la migración. Esto es claro cuando nos fijamos en la comunidad japonesa, cuyos integrantes son enviados en su mayoría por una empresa de su país, con objetivos organizacionales muy delimitados y periodos estrictamente predeterminados, lo que impide una cotidiana interacción con la 
sociedad más amplia en la que se insertan temporalmente y, en consecuencia, que no aprendan el rasgo cultural imprescindible para iniciar un proceso de aculturación que supone el idioma.

Frente a la respuesta adaptativa marcada por la segregación en el caso japonés, en el caso español la existencia de una comunidad de migrantes que conviven y reproducen sus pautas culturales no obstaculiza su integración con la sociedad regiomontana, con cuyos miembros también interactúan cotidianamente y de los que adquieren ciertas pautas culturales (eso sí, compatibles con su cultura de origen). El resultado es que se da en ellos una inmersión cultural relativamente mayor en la sociedad local.

Contrastando con estas diferencias, ambos colectivos de migrantes presentan coincidencias en sus dimensiones numéricas, en el estatus socioeconómico de la mayoría de sus miembros y en el importante peso relativo que tiene el sector de migración de carácter empresarial-trasnacional. Es por ello que consideramos pertinente volver a considerarlas para extender nuestro análisis comparativo a factores ligados a la cultura y a las prácticas empresariales.

\section{Espacios trasnacionales, expatriación y cultura empresarial nacional}

Hace casi tres décadas, Findlay y Gould (1989) señalaban la importancia creciente de la migración calificada y el peso, igualmente creciente, que en ella tenía la transferencia de personal dentro de empresas trasnacionales. La investigación acumulada desde entonces confirma tales impresiones ${ }^{4}$ (Findlay y Cranston, 2015) y muestra que la presencia de expatriados empresariales puede impactar significativamente las sociedades en las que residen (Beaverstock, 20II; Chung, 20I4). Nuestro interés se centra en un ámbito específico de esos posibles impactos: el influjo de estos expatriados en la cohesión de su comunidad de connacionales residentes en sus lugares de asignación. Para abordarlo, adoptamos una aproximación que integra elementos de la perspectiva administrativa sobre la expatriación, dentro de un más amplio enfoque trasnacional de los procesos migratorios. Es decir, exploramos cómo la configuración de los espacios sociales de una comunidad extranjera en un

4 Aunque debe reconocerse que el panorama actual de la movilidad de estos perfiles profesionales es más complejo que la mera circulación intraorganizacional (Mendoza, 2018). 
contexto local específico es condicionada por prácticas de administración de recursos humanos de las empresas de su nacionalidad que envían expatriados a sus filiales en ese espacio geográfico.

El marco referencial trasnacional se desarrolla al constatar que la situación de ciertos migrantes internacionales no puede ser comprendida como una simple adaptación a la sociedad receptora (Portes et al., 2002; Beltrán y Sáiz, 2009). Tales migrantes priorizan la construcción de "espacios sociales que vinculan sus países de origen y sus países de residencia" a través de "múltiples relaciones familiares, económicas, sociales, organizacionales, religiosas y políticas que traspasan las fronteras" (Glick, Basch y Blanc-Szanton, 1992: I). Faist (2000) distingue tres lógicas que determinan esas relaciones, vinculadas respectivamente a tres tipos de espacios trasnacionales: relaciones de reciprocidad en espacios de parentesco trasnacional; relaciones de intercambio en circuitos trasnacionales y relaciones de solidaridad en comunidades trasnacionales.

Estas distinciones nos permiten diferenciar entre circuitos trasnacionales empresariales y comunidades trasnacionales de connacionales migrantes, ${ }^{5}$ y a la vez plantear como hipótesis que, dada la relevancia de los expatriados empresariales en ciertas comunidades de extranjeros, ambos tipos de espacios trasnacionales y sus lógicas relacionales pueden interconectarse. Así, en la experiencia social de la expatriación temporal resulta fundamental poder construir espacios comunitarios de relación con connacionales; es decir, "emplazamientos sociales de origen extranjero enclavados en contextos locales" (Chung, 20I4: 8). Por otra parte, la construcción de esos espacios comunitarios podría estar condicionada por vínculos más formales de los expatriados con sus empresas nacionales.

En otro momento desarrollamos una amplia discusión sobre la forma de conceptualizar la cohesión grupal de las comunidades extranjeras (Doncel, 2013; 20I5a), por lo que ahora ofrecemos una síntesis. Partimos de la distinción entre un vínculo social más pragmático y una implicación grupal más íntima, como la que se establece entre socialidad y comunidad (Heller, 2002) (conceptualización estructuralmente análoga a la distinción entre circuitos y comunidades trasnacionales). La mayor cohesión la identificamos con la presencia de interacciones que denotan una "tendencia del

5 Distinciones especialmente relevantes, pues dentro del enfoque trasnacional se ha privilegiado el estudio de redes migratorias de carácter más informal. Sin embargo, paulatinamente se ha abordado desde esa perspectiva la situación más formalizada de los expatriados empresariales y a ello se ha invitado desde lo institucional (огм, 20I0) y la academia (Mendoza et al., 2016: 6). 
grupo en mantenerse y permanecer unido en la búsqueda de objetivos instrumentales o para la satisfacción de necesidades afectivas de los miembros" (Carron, Brawley y Widmeyer, 1998: 213). No obstante, ampliamos y enriquecemos esta concepción de la cohesión grupal con el término simmeliano de "sociabilidad", entendida como "forma lúdica de socialización" (Simmel, 2003: 8I) que trasciende los meros intereses materiales y pragmáticos de la interacción social.

A partir de esta conceptualización sobre cohesión grupal y tras la evidencia empírica generada en la fase previa del proyecto se propuso, como uno de los indicadores de esta cohesión (en su polo más pragmático), el grado de concentración en torno a ámbitos profesionales y/o a lugares específicos de trabajo. ${ }^{6} \mathrm{Sin}$ embargo, concentrarnos ahora en la influencia de los expatriados empresariales en la comunidad permite profundizar en el papel que juegan los lazos económico-organizacionales en la construcción del espacio social trasnacional. Por lo tanto, resulta necesario identificar componentes más específicos de la situación laboral-migratoria del expatriado que inciden en las dinámicas comunitarias. Para ello, nos apoyamos en aportaciones de la perspectiva administrativa de la expatriación.

Desde la perspectiva administrativa, las corporaciones trasnacionales deben gestionar sus recursos humanos y sus sedes con una visión global. Una de las decisiones más relevantes consiste en cuándo y bajo qué condiciones enviar temporalmente a sus filiales extranjeras personal técnico especializado o directivo desde sus matrices; sea porque resulta menos costoso (Bonache y $\mathrm{Pla}, 2002$ ) o para asegurar la lealtad y la reproducción de la cultura organizacional (Peixoto, 200I). Adicionalmente, debe decidirse sobre los incentivos económicos y los diversos apoyos que se brindan "para 'lubricar' los movimientos migratorios" del personal (Peixoto, 200I: I04I), sobre el grado de control de esos expatriados o la autonomía que se les permite (Harris y Holden, 200I) y sobre las formas como se buscará fomentar su adaptación para evitar su regreso anticipado. En principio, tales decisiones responden a criterios operativos racionales, pero pueden ser fuertemente condicionadas por la cultura de la organización, y esta, a su vez, por la nacionalidad de la empresa.

La noción de cultura empresarial nacional se ha utilizado ampliamente para referirse a valores y creencias compartidos en el mundo laboral y corporativo de

6 Los otros fueron: $a$ ) existencia de asociaciones o instituciones aglutinadoras de compatriotas; $b$ ) existencia y frecuencia de lugares y tiempos de reunión de connacionales; $c$ ) existencia de redes de ayuda (más informales) y d) grado de concentración de lugares de residencia. 
un país. A pesar de las justificadas críticas que ha recibido, ${ }^{7}$ es precisamente en relación tanto con la designación de directivos expatriados o locales para las filiales extranjeras como con las formas de control sobre ese personal donde se han identificado patrones diferenciados por país de origen entre las empresas trasnacionales. Ya Findlay y Gould señalaban, en ese sentido, la "enorme variación [...] entre compañías de distintos orígenes nacionales" (1989: 10). Siguiendo la tipología de Perlmutter (1969), las empresas japonesas suelen ser marcadamente etnocéntricas: asignan siempre directivos nacionales, replican sus prácticas organizacionales en el extranjero y ejercen un estricto control. Las europeas, en cambio, tienden a un etnocentrismo atenuado: prefieren asignar directivos nacionales, pero dan cabida a sus cuadros internacionales y ejercen un control menos rígido sobre las filiales ${ }^{8}$ (Peixoto, 200I; Bonache y Pla, 2002).

Más concretamente, pueden presentarse diferencias por nacionalidad de la empresa en la rigidez de las trayectorias de movilidad internacional de su personal, en la lealtad exigida hacia la compañía y en el grado de imposición de valores organizacionales en sus sedes extranjeras. También pueden variar las prestaciones y los apoyos que la empresa brinda a sus empleados para su instalación y vida cotidiana en el lugar asignado, entendidos como expresión de un control empresarial que puede darse tanto por vías formales como informales (Mirabal y Zapata, 2009). Adicionalmente, pueden presentarse políticas explícitas sobre la socialización del expatriado fuera de la empresa. Pero, independientemente de estas, las condiciones operativas antes mencionadas pueden condicionar las vidas personales de los empleados, y estas, a su vez, repercutir en sus dinámicas comunitarias más allá del ámbito laboral-empresarial.

\section{Metodología}

Aunque el proyecto inicial se realizó con una metodología mixta, para este trabajo retomamos únicamente los datos cualitativos generados. Estos se obtuvieron por

7 Los trabajos de Hofstede (1980) -su formulación más influyente- han sido cuestionados metodológica y epistemológicamente (McSweeney, 2002), lo mismo que su potencial para explicar nuevos hallazgos empíricos (Hayton et al., 2002).

8 Asimismo, el mundo empresarial japonés ha desarrollado un robusto corpus teórico-administrativo sobre gestión global, mientras que su contraparte española, solo tras el reciente crecimiento de sus inversiones en el exterior, ha comenzado a reflexionar sobre el tema. 
medio de una aproximación etnográfica que incluyó la observación participante en eventos de cada comunidad nacional migrante, así como entrevistas en profundidad con migrantes y otros informantes clave contactados a través de la técnica de bola de nieve. Para los casos de los dos países seleccionados nos concentramos en las entrevistas en profundidad con informantes clave y miembros de la comunidad más relevantes por su conocimiento y cercanía de la situación de los expatriados empresariales. Se realizaron ocho entrevistas para el caso español y cinco para el japonés.

\section{Cuadro i}

Informantes sobre la comunidad española.

\begin{tabular}{lllll}
\hline$N$ & Identificación & Género & Edad & Lugar de origen \\
\hline I & Cónsul de España en Monterrey & Masculino & 44 años & Granada \\
\hline 2 & $\begin{array}{l}\text { Técnico de alto nivel expatriado por } \\
\text { empresa española }\end{array}$ & Masculino & 52 años & Córdoba \\
\hline 3 & $\begin{array}{l}\text { Técnico de alto nivel expatriado por } \\
\text { empresa española }\end{array}$ & Masculino & 34 años & Cartagena \\
\hline 4 & $\begin{array}{l}\text { Ama de casa (casada con empleado de } \\
\text { compañía mexicana con presencia de } \\
\text { expatriados españoles) }\end{array}$ & Femenino & 58 años & San Sebastián \\
\hline 5 & Presidente asociación Euromex & Masculino & 53 años & Madrid \\
\hline 6 & Profesor universitario & Masculino & 37 años & Madrid \\
\hline 7 & $\begin{array}{l}\text { Dueño de restaurante de comida espa- } \\
\text { nola }\end{array}$ & Masculino & 55 años & Pamplona \\
\hline 8 & $\begin{array}{l}\text { Presidente del Centro Asturiano en } \\
\text { Monterrey }\end{array}$ & Masculino & 55 años & Oviedo \\
\hline
\end{tabular}

Fuente: Elaboración propia con base en el operativo de campo

Cuadro 2.

Informantes sobre la comunidad japonesa.

\begin{tabular}{lllll}
\hline$N$ & Identificación & Género & Edad & Lugar de origen \\
\hline 9 & $\begin{array}{l}\text { Cónsul honorario de Japón en Monterrey } \\
\text { (nacionalidad mexicana) }\end{array}$ & Masculino & 57 años & Monterrey \\
\hline Io & $\begin{array}{l}\text { Técnico de alto nivel expatriado por } \\
\text { empresa japonesa }\end{array}$ & Masculino & 45 años & (sin dato) \\
\hline
\end{tabular}




\begin{tabular}{lllll}
\hline II & $\begin{array}{l}\text { Dueño de un restaurante de comida } \\
\text { japonesa }\end{array}$ & Masculino & 6I años & Kagoshima \\
\hline I2 & $\begin{array}{l}\text { Dueña de restaurante de comida japo- } \\
\text { nesa }\end{array}$ & Femenino & 39 años & Tokio \\
\hline I3 & Antropólogo e investigador & Masculino & 35 años & Totori \\
\hline
\end{tabular}

Fuente: Elaboración propia con base en el operativo de campo

En la distribución de características de los informantes se buscó un equilibrio que arrojara luz acerca del influjo de expatriados sobre las dinámicas comunitarias correspondientes atendiendo a las características de estas comunidades. Así, la muestra de informantes españoles representa la heterogeneidad laboral y ocupacional que caracteriza a su comunidad en Monterrey. En el caso japonés, si bien solo contamos con un informante que podamos considerar expatriado empresarial, es importante señalar que tanto el cónsul honorario de Japón (inf. 9) como el antropólogo e investigador japonés (inf. 13), actuaron en calidad de informantes clave. El primero de ellos, por tratarse de un empresario cuyo conocimiento de la comunidad radica en su constante interacción con expatriados japoneses en el espacio empresarial. El segundo, por haber realizado un amplio trabajo etnográfico sobre los expatriados japoneses durante el cual ha entrevistado a numerosos expatriados con quienes también ha convivido cercanamente, $y$ ha actuado como mediador intercultural en varias empresas japonesas radicadas en Monterrey. Además, este informante también colaboró como investigador en el proyecto inicial, con el estudio de la comunidad japonesa. Asimismo, el trabajo académico de este informante (Hirai, 2013; 20I5), asentado sobre su abundante trabajo de campo y revisión documental (de información empresarial estadística y de manuales de administración japoneses), nos acercó a la comprensión de las políticas generales de las empresas japonesas. En todo caso, de estas políticas nos interesa destacar la dimensión vivencial y subjetiva que de ellas hace el expatriado.

La información proporcionada por estos entrevistados y recuperada de las fuentes secundarias señaladas se analizó en busca de patrones de interacción grupal de los expatriados empresariales con otros connacionales (expatriados o no), así como de condiciones laborales y de respaldo empresarial que tienen incidencia directa o indirecta en tales interacciones. ${ }^{9}$ Ciertamente, las diferencias en la distancia cultural de cada nacionalidad con respecto a la sociedad receptora, y la escasa presencia

9

La consideración del tamaño y otras características de las empresas no fueron tomados en cuenta por encontrarnos en un acercamiento exploratorio que no queremos saturar 
femenina entre nuestros informantes, limitan las posibilidades de generalización de los resultados obtenidos. Sin embargo, siendo un estudio exploratorio permite extraer sugerentes conclusiones preliminares y abre nuevas líneas de reflexión e investigación.

\section{Los expatriados empresariales en las comunidades española y japonesa en el AMM}

En la fase previa del proyecto se consideraron miembros de "comunidades extranjeras" aquellos que, además de pertenecer a alguna nacionalidad seleccionada, hubieran nacido y vivido en forma ininterrumpida al menos I5 años en su país de origen. Esos criterios delimitan el marco comunitario dentro del cual exploramos ahora el influjo particular de los expatriados empresariales, entendidos estos como aquellos directivos y técnicos de alto nivel asignados por empresas con matrices en su país de origen para laborar temporalmente (generalmente entre dos y cinco años) en el AMM.

En 2009, el registro de migrantes españoles en Nuevo León variaba entre 612 y 80o, según el Instituto Nacional de Migración (INM) (Rodríguez y Cobo, 20I2) y el consulado español, respectivamente. Entre ellos, los informantes identifican, además de los expatriados por empresas españolas, a altos mandos en empresas mexicanas, empresarios, religiosos, académicos universitarios, y una oleada reciente de profesionistas independientes. En el trabajo de campo también localizamos, excepcionalmente, trabajadores de estatus socioeconómico bajo. A pesar de esta heterogeneidad ocupacional y de no ser mayoría, los expatriados empresariales constituyen (junto a sus compatriotas contratados por empresas mexicanas) una de sus caras más visibles: "La mayoría [de españoles] que viene aquí es porque vienen de empresa" (inf. 7). En cuanto al género, esta comunidad es cuantitativamente equilibrada. Si bien la asignación de empleados al extranjero en las empresas españolas es casi completamente masculina, generalmente les acompañan sus parejas y familias.

Por contraste, el colectivo de japoneses en Nuevo León es altamente homogéneo. De los 478 que según el InM residían en el estado en 2009 (Rodríguez y Cobo, 20I2), el cónsul honorario de Japón (inf. 9) calculaba unos 300 expatriados empresariales. Esto resulta congruente con la presencia de 67 empresas japonesas en Nuevo León en 20II (Hirai, 2013), pues cada una tiene al menos tres empleados de su país. Los

prematuramente de variables explicativas. Sin embargo, asumimos que estos y otros criterios abrirán nuevas y promisorias vías de análisis. 
japoneses no ligados al mundo de la empresa son una minoría marginal. Adicionalmente a esta elevada proporción numérica, el expatriado empresarial (hombres exclusivamente) resulta central en la imagen de la comunidad completa en el AMM de la mayoría de los informantes, constituyendo entonces una migración eminentemente masculinizada.

Los migrantes españoles ligados al mundo de la empresa y sus familias han consolidado un activo núcleo comunitario. De ellos, son unos pocos empresarios independientes, así como españoles traídos por empresas mexicanas de forma permanente, quienes, por su mejor conocimiento del contexto local y su mayor capital social, funcionan como nodos de conexión de la comunidad. ${ }^{10}$ De hecho, la trasnacional mexicana $\operatorname{CEMEX}^{11}$ representa un referente colectivo central (inf. I y 4 ).

Sin embargo, los expatriados temporales constituyen la mayor parte de este colectivo (entre 30 y 40 familias), tratándose de un grupo homogéneo en estatus profesional y social, que no extiende la solidaridad a otro perfil de migrantes ni busca abiertamente una reafirmación identitaria nacional. De ahí su distanciamiento de organizaciones como el Centro Asturiano (abierto a todos los perfiles socioeconómicos e incluso a mexicanos descendientes de españoles). "Ese tipo de español (expatriado) no es fácil que se asocie”, confirma el presidente de esta asociación (inf. 8), ${ }^{12}$ lo que el cónsul explica por la ausencia del deseo de estar "españoleando todo el día" (inf. I). El grupo de expatriados tampoco se vincula con redes que procuran el apoyo entre españoles más allá de los círculos sociales cercanos, y no tienen conocimiento de iniciativas como Españoles en Monterrey, una página de Facebook creada con ese fin, muy popular entre otros sectores de la comunidad. Dado que las necesidades materiales son poco apremiantes para ellos, con excepción de una parte de los profesionistas independientes, ${ }^{13}$ su solidaridad tiene un carácter más lúdico.

${ }^{10}$ Sobrepasa los objetivos de este trabajo analizar con mayor profundidad cómo las diferencias históricas de la presencia de ambas comunidades extranjeras en el AMM condicionan la construcción de sus espacios sociales, aunque evidentemente el mayor arraigo de ciudadanos españoles favorece a ese colectivo.

${ }^{11}$ Esta cementera mexicana, tras absorber a dos empresas españolas en 1992, incorporó a directivos de estas en sus oficinas centrales en Nuevo León.

12 Véase la figura I, donde estas distinciones entre subgrupos dentro del colectivo nacional y con respecto a la sociedad receptora son marcadas como círculos continuos, si la distinción es tajante; o punteados, si es más permeable. Igualmente se indican con breves líneas rectas las relaciones puntuales que traspasan los límites entre círculos o ponen en contacto a dos de ellos.

${ }^{13}$ Estos, sin embargo, son considerados "unos cuantos que están en la comunidad y que 
Este grupo de españoles "de empresa" (expatriados y no expatriados) organiza esporádicamente reuniones en las que se incluyen paisanos de perfil socioeconómico similar al suyo, como son profesionistas migrados sin apoyo empresarial y académicos adscritos a universidades locales. Estos encuentros, sin embargo, no impactan profundamente en la configuración de sus espacios sociales. Uno de los académicos que ha asistido señaló al respecto que, aunque "se tiene la oportunidad de conocer a mucha gente [...] A mí personalmente se me hace muy curioso para tener una relación muy cercana, porque los intereses son diferentes" (inf. 6). Un expatriado reconoce además que esos esfuerzos por reunir a más compatriotas no implican una afinidad real; así, por ejemplo, la posada navideña que organizan se hace "con mucha dificultad, porque hay mucho de ay, esto no me gusta, con aquel discutí, este es un pesado, este es un engreído, este es un chulo"' (inf. 2).

\section{Figura I.}

Configuración comunitaria de las migraciones española y japonesa

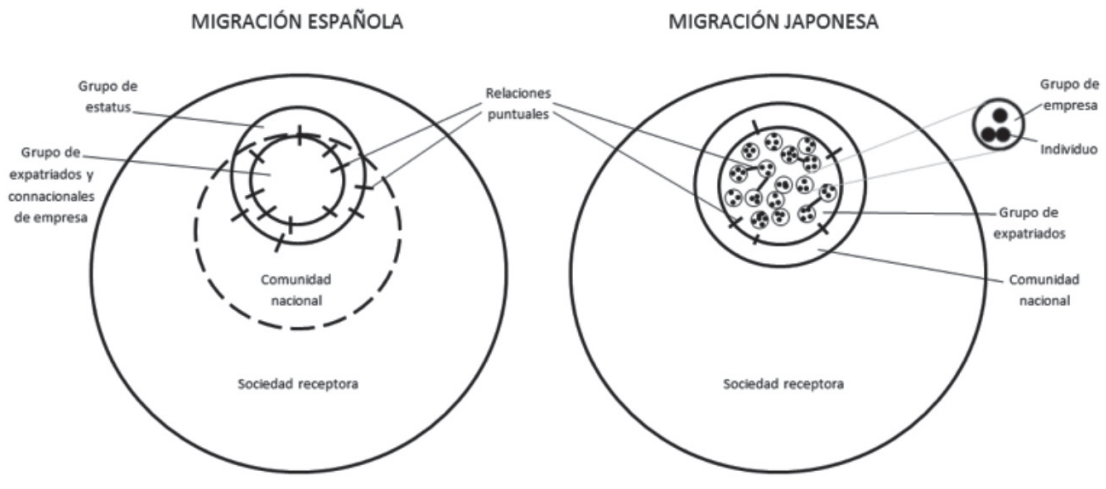

Fuente: Elaboración propia

El perfil predominante del japonés en el Амм es el de hombres de una elite empresarial, entre los 30 y los 55 años de edad. Solamente a unos pocos los acompañan

están mucho más jodidos [...] porque tienen menos dinero para vivienda, menos dinero para vehículos y algunos pasan muchos apuros". El apoyo a ellos, se subraya, es "por (considerarlos) amigos, más que por comunidad, pues se les apoya en parte. No en dinero pero sí en, no sé, en cederle puntos para viajar a España (por ejemplo)" (Inf. 2). Como podemos ver, debido al alto estatus socioeconómico de este sector de la comunidad, al apoyo económico directo se considera como tabú. 
sus familias. La homogeneidad de esta población propicia la identificación entre los individuos que la conforman y su aislamiento del contexto social más amplio. Pero ello no propicia una intensa vida grupal, sino que "sus vidas migratorias [tienden a ser] monótonas, rutinarias y solitarias" (Hirai, 2015ः II8).

El primer año se caracteriza por mucha soledad [y] el aislamiento de la sociedad receptora fuera del trabajo [...] su círculo social no se desarrolla tan fácilmente más allá de las relaciones sociales que han establecido con pequeños grupos de japoneses de sus compañías o de otras a través del trabajo [...] la mayor parte del tiempo libre [lo] pasan en sus casas o salen con pequeños grupos de amigos o compañeros del trabajo para cenar (Hirai, 2015: II7-II8).

Otras actividades de esparcimiento procuradas por estos expatriados, por ejemplo, el golf y el karaoke (inf. 9), confirman que el colectivo de japoneses tiende a la atomización en pequeños grupos. Además, su distanciamiento de los pocos connacionales ajenos al mundo de la empresa es casi absoluto. Igualmente evitan vincularse con la activa comunidad Nikkei, ${ }^{14}$ a pesar de que el embajador buscara propiciar ese acercamiento (inf. 9). Así, "la interacción social con sus compatriotas y compañeros de trabajo se convierte, casi obligadamente, en la única interacción social posible" (Doncel, 2015a: 158), en gran medida por rasgos de la cultura empresarial japonesa sobre los que profundizaremos más adelante.

A pesar de ello algunas prácticas sociales aminoran ese aislamiento y dan al colectivo un mínimo grado de comunitarismo. Los pocos japoneses acompañados de sus familias se reúnen los sábados en un colegio donde sus hijos reciben clases en japonés y en algunas ocasiones realizan actividades deportivas (inf. 9, IO, II). Para los expatriados sin familia, el deporte ha sido también el medio más efectivo para ampliar sus espacios sociales más allá del eje laboral. La conformación de un equipo de futbol que compite en una liga local ha propiciado la convivencia entre empleados de diferentes compañías y, en menor medida, ha contribuido a "estrechar los límites que separan a la comunidad de la sociedad de acogida" (Doncel 2015a; 16I), dado que integra a mexicanos. También se realizan, aunque menos frecuentemente, torneos de softbol entre equipos de diferentes compañías.

${ }^{14}$ Descendientes de japoneses emigrados. 


\section{Cultura empresarial, etnocentrismo y trayectorias de expatria- ción-repatriación}

Generalmente, las trasnacionales japonesas han desarrollado estrictos lineamientos para la administración de sus sedes extranjeras. La asignación del personal japonés a cargos directivos en estas sedes"se ha convertido en un elemento fundamental para las estrategias de formación de recursos humanos y administración global" (Hirai, 20I5: II2). Una de sus principales motivaciones es asegurar la lealtad a la empresa y que la gestión de las filiales se ajuste a la cultura organizacional de la matriz. Este patrón intensamente etnocéntrico se confirma en las empresas japonesas instaladas en Monterrey que "tienen a ciertas personas clave de Japón [... ] dos o tres personas clave, nada más, el resto son mexicanos" (inf. 9). Igualmente, ejercen un control estricto sobre ellos (por ejemplo, deben comunicarse en horarios laborales de Japón, lo que dificulta su adaptación al contexto local) que los aísla del resto del personal y los mantiene más vinculados, incluso emocionalmente, a la oficina central (inf. I3).

Asimismo, la exigencia de una entrega profunda a los intereses de la compañía es propia de la cultura empresarial japonesa (inf. 9, II, I3)."Del cien por ciento del tiempo [...] que están aquí en Monterrey deben de pasar el noventa por ciento del tiempo en el trabajo [...] Nada de actividades sociales", comenta el cónsul honorario (inf. 9). Esta demanda de lealtad desalienta, incluso, la interacción con japoneses de otras compañías con quienes los encuentros sin una finalidad netamente laboral son casi nulos. Nuestro principal informante japonés da una interpretación de tal fenómeno a raíz de la siguiente anécdota:

[Un japonés recién llegado a México] iba a entrar al equipo de futbol de los japoneses pero su jefe le dijo que no debe de tener tanta amistad con esos paisanos [...] porque si él hace algo más y captura la amistad, la relación entre su compañía con otras se vuelve un poco complicada. Por eso le dijo que mantuviera la distancia (inf. 13).

Congruentemente con esta lealtad demandada, la expatriación es un paso en una trayectoria laboral internacional rígidamente predefinida. El empleado que ingresa en ella generalmente tiene dos o tres periodos de trabajo en el exterior, intercalados con regresos a Japón. Cada desplazamiento supone un ascenso en sus responsabilidades y en su jerarquía en la empresa.

Las firmas españolas, por su parte, han desarrollado en los últimos años estrategias y procedimientos para la asignación al extranjero de su personal, pero su situación resulta más flexible. En primer término, la salida al exterior no necesaria- 
mente supone un ascenso ni se inserta en una trayectoria estrictamente predefinida. Mientras la primera salida de un japonés puede representar pasar de su vida austera en Japón a gozar de lujos como piscina y vigilancia privadas (Hirai, 2013: 92), la percepción subjetiva del expatriado español es que mantiene el nivel de vida que tenía en España y que, más que un paso claramente ascendente en su formación, te eligen "porque has hecho una carrera y porque tienes un prestigio [...] [Por lo tanto], no hay mejora [económica, pero] no hay tampoco escatimación [...] por parte de la empresa" (inf. 2).

El apego a una cultura organizacional propia en las operaciones en el extranjero tampoco es percibido tan estricto por los expatriados españoles como ocurre entre los informantes japoneses. Observamos, de hecho, un lento pero progresivo paso de una gestión etnocéntrica a una policéntrica. Muestra de ello es un caso en el que para algunos puestos directivos "la empresa está procurando buscar mexicanos de buen talante [... ] de confianza para la empresa" (inf. 2). Asimismo, aunque la lealtad a la compañía es uno de los fines de la expatriación de empleados, esta no se exige tan intensamente. Así, un expatriado habló de su oposición a un traslado ordenado por la empresa de la siguiente manera: "Me costó que varios de mis jefes ya estén muy cabreados conmigo. [Pero] no es obligación, no es ley, tienes el derecho de que no te pase nada por negarte" (inf. 2). Situación que contrasta con lo que describe el antropólogo japonés: "cuando trabajas para una empresa japonesa tienes que entrar a una etapa de socialización y apropiación de valores tradicionales, tienes que respetar al jefe [... ] entonces, el muchacho japonés va cambiando [se adapta a] la vida empresarial, machista y dominante, elitista" (inf. 13).

En cuanto a la duración de las estancias, si bien para ambas nacionalidades se ajusta a los periodos comunes a este tipo de migración ( $2-5$ años), pudimos constatar variaciones en la rigidez de las trayectorias de movilidad que tienen un fuerte influjo en la situación social de los expatriados. Las estancias de los japoneses se encuentran programadas con precisión y sin ninguna flexibilidad. Su duración depende de la fase en que se encuentra la carrera del empleado y, por lo tanto, da lugar a un patrón por grupos etarios. Hirai proporciona una detallada descripción.

Antes o después de cumplir 30 años de edad [...] reciben el nombramiento de trabajar en el extranjero por primera vez para recibir capacitación u ocupar un puesto menor [...] durante dos años. Después [...] regresan a las compañías matrices en Japón, y posteriormente reciben el nombramiento de dar servicio en otros países [que] dura alrededor de tres años, pero ocupan puestos intermedios y recibiendo misiones más importantes. Terminando el segundo servicio en el extranjero nuevamente regresan 
a Japón [... U. Unos años después de cumplir 40 años de edad, algunos [... salen de Japón en la tercera ocasión, ahora para ocupar el puesto de presidente o director general de las subsidiarias locales o regionales. En esta ocasión, su estancia en el extranjero dura cinco años (Hirai, 2015: 113).

Todos los entrevistados perciben la brevedad de la estancia como un rasgo distintivo de la migración empresarial japonesa. Según el cónsul honorario, "la comunidad es muy volátil. Vienen y se van" (inf. 9). Especialmente en las primeras salidas al extranjero esto desalienta tanto la adaptación a la sociedad receptora como la construcción de redes sociales de connacionales más intensas. Determina, asimismo, que el expatriado se traslade generalmente sin su familia, siendo así uno de los principales factores de la soledad de la experiencia migratoria japonesa antes referida.

Además, como vemos en la cita anterior, la trayectoria de los expatriados japoneses les hace retornar a Japón constantemente, lo cual orilla a los pocos expatriados que se trasladan con hijos a brindarles educación que les permita reintegrarse al sistema educativo japonés; como veremos, este es uno de los pocos motivos de sociabilidad inducidos indirectamente por la cultura empresarial japonesa.

Las empresas españolas con presencia en Nuevo León también determinan estancias breves para sus expatriados, pero, según el cónsul, resulta común que se extiendan año con año (inf. I). Esta apreciación es confirmada por la experiencia previa de los expatriados españoles entrevistados, quienes anticipan que pueden ser requeridos por más tiempo del acordado inicialmente (inf. 2 y 3). También es frecuente que los españoles sean trasladados entre diferentes locaciones extranjeras sin pasar por su país de origen. La relativa incertidumbre sobre la trayectoria y el tiempo de permanencia en el extranjero propicia que al expatriado español, por lo general, lo acompañe su familia. Esta mayor incertidumbre y, particularmente, la presencia de las mujeres que acompañan como parejas a los expatriados, veremos más adelante, intensifican las posibilidades de interacción grupal. Adicionalmente, la significativa presencia de los españoles traídos por empresas mexicanas imprime al colectivo de españoles de empresa una liga más laxa con la cultura empresarial de su país de origen.

\section{Respaldo y control empresarial}

Como puede inferirse a partir de lo presentado hasta ahora, la cultura empresarial nacional determina el grado de apoyo y control ejercido sobre las condiciones de vida de los expatriados. Para el caso de los españoles, el apoyo se expresa en asig- 
naciones económicas reguladas con precisión, destinadas a su traslado, alquiler de vivienda, la educación de sus hijos y viajes a España. Sin embargo, la empresa no brinda orientación en la búsqueda de cubrir tales necesidades en el Aмм. ${ }^{15}$ Por ello, particularmente en la primera asignación internacional, se recurre al consejo de otros españoles asentados previamente (inf. 2 y 3 ), de tal modo que la limitación de la injerencia empresarial incentiva la búsqueda de connacionales más allá del espacio laboral.

Además, este respaldo mediante asignaciones económicas especiales puede ser más limitado para algunos empleados que ingresan en la compañía al momento de su asignación internacional y, por ello, son contratados directamente por la filial mexicana. Al respecto, un expatriado español comentó: "Son diferentes condiciones económicas. No tienes derecho a tanto viaje, el valor de la casa es casi que "busca la casa a ver cuánto te cuesta y ya veré lo que te doy', [incluso] el sueldo es menor" (inf. 2). Tales condiciones, evidentemente, incentivan más la búsqueda del apoyo de otros connacionales.

Como mencionamos antes, las compañías japonesas suelen trasladar al extranjero solamente a personal de elite (o en camino a convertirse en tal), al que ofrecen un amplio respaldo económico. Ello, aunado a las dificultades idiomáticas y al control que busca ejercerse, orilla a las empresas a encargarse en gran medida de las cuestiones prácticas para la instalación y la estancia de sus expatriados. Esto se observa claramente con respecto a la residencia de sus empleados. Para ambas nacionalidades, San Pedro es el municipio de mayor concentración de expatriados, por ser el más seguro y exclusivo del AmM. Sin embargo, los españoles pueden elegir su vivienda (siempre que se ubique en ese municipio u otras zonas de estatus similar), por lo que tienden a vivir cercanos entre sí sin necesariamente ser vecinos (inf. 3). En cambio, los japoneses "deben respetar el lugar que les asigna la empresa [para vivir]" (inf. 13), ubicado invariablemente en San Pedro. Así, varios japoneses pueden residir en los mismos condominios o en domicilios muy cercanos. Pero esta congregación forzada en pequeños núcleos de individuos no da lugar a una cohesión comunitaria, sino al panorama de atomización que representamos visualmente en la figura I.

Con el pequeño grupo de compañeros japoneses de la empresa se va rutinariamente de compras o a cenar, por ejemplo, y grupos de directores de diferentes compañías se reúnen periódicamente en restaurantes japoneses para tratar asuntos de negocios (inf. II). Así, otro de los rasgos que caracterizan a las empresas nipo-

${ }^{15}$ Con excepción de limitar los lugares de residencia, que comentaremos de forma especial dado el impacto que tiene en las dinámicas intracomunitarias. 
nas instaladas en el Amm es el acaparamiento de espacios y actividades sociales no laborales de sus expatriados. Incluso la asistencia a los table dance puede ser una actividad laboral, nuevamente, una situación que responde a la cultura empresarial nacional. En palabras del informante que lo refirió,"los cabarets o clubes nocturnos en Japón son muy importantes. Prácticamente es un requisito con la negociación con los clientes. Aquí muchos empresarios van a los table dance con los clientes [... ] y son cubiertos por los gastos de la empresa" (inf. I3).

Por otra parte, ciertas prácticas de ocio son absorbidas por los espacios laborales físicos, lo cual, una vez más, obstaculiza el surgimiento de redes comunitarias más extensas. Un expatriado confirmó que uno de sus principales puntos de encuentro con otros japoneses eran "las celebraciones dentro de la empresa" (inf. Io), mientras que el cónsul honorario compartió que los directivos japoneses en ocasiones se reúnen en su oficina a tomar una copa (inf. 9). Los expatriados españoles, por su parte, no mencionaron que la empresa busque influir en sus dinámicas sociales no laborales. Solamente un informante refirió que, durante un periodo, el consulado y algunas empresas españolas convocaron a reuniones sociales entre empresarios, expatriados y académicos, pero tuvieron poco impacto y se abandonaron (inf. 6).

Otras diferencias en el control empresarial entre ambos países se observan en la injerencia en las actividades extralaborales cotidianas de los empleados y sus familias; injerencia condicionada por la inseguridad que afecta a México. Las empresas españolas han aconsejado a sus empleados no viajar por el país. Por su parte, las japonesas han llegado a prohibirlo. Durante algunos periodos también han prohibido salir del municipio de San Pedro, e incluso de su domicilio, en los tiempos de ocio. Igualmente, en algunos momentos las empresas determinaron el regreso a Japón de familias de expatriados (inf. 13). Al respecto, un expatriado español comentó también con detalle un protocolo de seguridad emitido por su empresa que, aunque riguroso, no es de observancia forzosa, por lo que él ha decidido no seguirlo:

Si lees el protocolo, todo lo que te dicen ahí que tienes que seguir, casi casi que estaría muerto de miedo [...] porque te cuentan: "no hables con desconocidos, no te bajes del carro sin mirar no sé qué, no entres a un Oxxo, no vayas a un mercado". Te dicen que no hagas nada. Hay personas y hay mujeres que aquí tienen miedo realmente y lo cumplen. Yo, como toda la vida he andado por todas partes del mundo y mi esposa ha ido conmigo siempre... y nos hemos movido por todas partes, pues mi esposa no lo cumple porque no lo quiere cumplir, porque ella quiere vivir a su aire y si le toca le tocó (inf. 2). 
Las empresas japonesas, por su parte, emiten prohibiciones similares que son obligatorias e interfieren directamente en la vida cotidiana de la familia de sus empleados, como la de usar el transporte público o, en algunos casos, que las esposas conduzcan automóvil (inf. 13). Otra diferencia que recae en las esposas regula su situación laboral. Algunas empresas españolas están obligadas a brindarles empleo a ellas si lo solicitan (inf. 2). Es una forma de procurar su bienestar emocional y de compensar el sacrificio profesional que pudo representarles abandonar España. En cambio,"la mujer japonesa no puede trabajar; las empresas donde trabaja el esposo lo prohíben" (inf. 13). Tales medidas, según nuestro informante antropólogo, se encuentran en sintonía con la cultura empresarial japonesa que "sacrifica demasiado la vida personal [pues el empleado] no puede tomar iniciativa y luego no puede hacer nada cuando no tiene respaldo de la organización" (inf. I3), y que, asimismo, promueve la dominación masculina (inf. 12 y 13). Todas estas limitaciones de movilidad y decisión, ejercidas en un entorno extraño para el expatriado, orillan al ostracismo social. De forma más significativa aún, la escasa presencia de mujeres y las limitaciones que les imponen obstaculizan la formación de redes de connacionales más intensas.

\section{Presencia de la familia y las mujeres}

Como hemos visto, la flexibilidad en la duración de la estancia y otros rasgos de la cultura empresarial española fomentan que los expatriados viajen con sus familias. Además, las asignaciones económicas son mayores en esos casos. Esto fomenta que la migración de expatriados españoles esté conformada principalmente por grupos familiares. Ya adelantamos que esta condición promueve una más intensa interacción social; particularmente por el rol que juegan en ello las mujeres. ${ }^{16}$

En los espacios sociales de españoles que construyen los expatriados, el papel de la mujer resulta fundamental. Son las esposas quienes establecen rutinas de convivencia, participan en redes de apoyo y organizan reuniones que permiten constatar una cohesión grupal que supera los límites del intercambio con miembros de la misma empresa e incluso el del círculo exclusivamente de expatriados. Así lo reconoce uno que compartió más detalles sobre esa dinámica grupal:

16 Existe una amplia investigación sobre la participación activa de la mujer en la migración calificada, pero continúa predominando su situación como parejas de hombres expatriados (Willis y Yeoh, 2002), lo que generalmente trunca su desarrollo profesional e intensifica roles tradicionalmente considerados "femeninos" (Ho, 2006), entre los que se destaca su carácter de agente socializador (Ortiz Guitart y Mendoza, 2007). 
Sí, sí [hay una red de españoles]. Pero son las esposas [... . Son ellas las que se organizan. Tienen más horas libres y son las que se organizan para todo. Los jueves en la mañana se juntan para desayunar casi todas las españolas. Por lo menos el grupo de mi esposa... Mi esposa queda casi todos los días para verse con las amigas para ir aquí o allá... cada día tienen un evento, ir a comprar al mercado central, o así [...] . Los viernes siempre cenamos juntos un grupo concreto, no muchísima gente, y luego casi todos los sábados o domingos siempre hay un evento en casa de alguien. Todas las semanas siempre hay tres o cuatro momentos, por lo menos las esposas siempre hay tres o cuatro momentos de reunión con españolas (inf. 2).

Comparativamente con otros núcleos de convivencia grupal de españoles, esta intensa interacción posee menos formalidad y no supone una solidaridad que incluya el apoyo material o económico. Puede entonces sostenerse que las relaciones en el núcleo grupal al que pertenecen los expatriados cubren una necesidad gregaria y afectiva. Aun así, además de cultivar la sociabilidad, pueden intercambiar información y compartir el capital social que algunos miembros del grupo han acumulado. Así podemos verlo en la siguiente cita, que si bien es extensa, adicionalmente subraya la feminización de esa integración, la forma en que la falta de orientación por parte de la empresa incentiva las dinámicas sociales y la inclusión de otros españoles de similar estatus socioeconómico.

Mi esposa si tiene cualquier problema inmediato, pues llama a las amigas, a las que llevan aquí más tiempo. La esposa de mi jefe... trabajó en el consulado de España hace I2 años [... Entonces ya conoce todo, desde hospitales y qué hacer y qué no hacer. Entonces siempre se busca consejo. Mi hijo, por ejemplo, la semana pasada se rompió un brazo y tuvieron que operarlo de emergencia [... . Pues llámalos [pregúntales] ¿a dónde me dirijo?, ¿[con] quién voy?, ¿quién es de fiar?, ¿quién no es de fiar? Y siempre te dan pistas, obviamente. Pero eso del apoyo buscamos más por el grupo, la empresa no apoya tanto. Quizás tengan mejor [información] los expatriados de otras empresas que la propia empresa. Los expatriados que llevan más tiempo aquí, que conocen, que saben y que nos apoyamos. De hecho, la tienda que está enfrente aquí, La castellana [... trae muchos productos de España, es de un español que es amigo mío que se vino aquí en el año 64 [... es un tipo que ya está muy bien situado económicamente [...] tiene un whatsapp para todas las conocidas y él dice "ya tengo en la tienda jamón, ya tengo en la tienda no sé qué..." y van todas. Igual te dice "si tienes un problema..." le hablamos "oye Pepe, ¿ [con] que médico me dirijo?” [...] Es un tipo que tiene contactos, que está muy bien situado aquí en Monterrey, además, 
su mujer es mexicana [... él se encarga de llamar al médico y le dice "oye, va a ir una española amiga que se llama tal, con este problema, atiéndela tal" y obviamente mucho mejor que llegar ahí sin nada, no puede ser de otra manera, hay que agarrarse a lo que tenemos (inf. 2).

La situación de los expatriados japoneses contrasta con este panorama. Tenemos un colectivo de "hombres de negocios [que] vienen ellos solos [y que] no tienen ninguna actividad social" (inf. 9). Si bien en la fase anterior de la investigación se había determinado un alto grado de cohesión intracomunitaria, en buena medida con base en el grado de concentración residencial y de lugares de trabajo, debemos subrayar nuevamente que se trata más bien de pequeños núcleos atomizados de expatriados con escasa interacción grupal en un espacio social más amplio. En esos núcleos se observa un acompañamiento rutinario inducido en parte por el control que sobre su movilidad ejerce la empresa, pero con poco espacio para el cultivo lúdico y afectivo de la sociabilidad.

Antes destacamos que la presencia limitada de familias y mujeres en la migración japonesa es condicionada por la brevedad de la estancia. Es preciso ahora considerar otras raíces culturales más profundas. La cultura empresarial de este país es, en la opinión de algunos miembros marginales de la comunidad japonesa en el AMM, heredera y continuadora de una cultura nipona jerárquica y patriarcal que tiende a limitar el papel de la mujer al de ama de casa y madre de familia (inf. I2 y I3). Por lo tanto, al no existir las condiciones en el Амм para desplegar una vida familiar japonesa tradicional (por el reducido volumen de población japonesa, la inseguridad y la relativamente mayor apertura a la igualdad de género), su presencia tendería a evitarse.

La excepción, en este caso, confirma la regla. Es a partir de la presencia de pocas esposas y familias de los expatriados que tiene lugar cierta dinámica comunitaria que integra a los pequeños núcleos atomizados que conforman la mayor parte de esta migración. Se trata de reuniones en un colegio que se alquila los sábados para que los hijos reciban clases en japonés, las cuales, antes mencionamos, dan pie a cierta interacción entre familias y a la organización esporádica de competencias deportivas (inf. 9, IO, II). Aun así, debemos insistir, es una dinámica de la que están excluidos la mayor parte de los expatriados. 


\section{Conclusiones}

Hemos ofrecido un análisis comparativo de dos grupos en una situación equiparable por su condición de migrantes, el tamaño de su población y la centralidad que en ellos tienen los expatriados empresariales. La homogeneidad ocupacional de los japoneses contrasta con la heterogeneidad de la de la comunidad española; no obstante, el grupo de expatriados españoles también se caracteriza por su homogeneidad. Así, ambos grupos coinciden en la barrera creada con el resto de la comunidad de connacionales (aunque en el caso español sí aparece cierta pulsión por superarla, el límite marcado por la coincidencia de estatus socioeconómico nunca es sobrepasado). En este punto planteamos la hipótesis de que la ostentación de cierto estatus es, en ambos casos, explicativa del aislamiento con respecto a la comunidad más amplia. Este aislamiento en el caso español limita incluso el involucramiento de los expatriados en extendidas dinámicas comunitarias, resultado de una arraigada presencia de españoles en México y en el AMM. Más allá de esta coincidencia, sus interacciones intracomunitarias ofrecen imágenes claramente diferenciadas. Por ejemplo, en el caso japonés, aparte de la segregación de un grupo de estatus respecto de los pocos japoneses que no pertenecen al mundo empresarial, dicho grupo se caracteriza por un alto grado de fragmentación interna en torno a cada una de las empresas para las que trabajan.

En cuanto se refiere al papel de las empresas y de las condiciones laborales que inciden en las dinámicas sociales, la rigidez y el marcado etnocentrismo organizacional característicos de las empresas japonesas contrasta con la mayor flexibilidad de las españolas y su etnocentrismo atenuado. Esta flexibilidad se deja sentir en diferentes ámbitos: en las condiciones dadas para el movimiento migratorio y en su relación laxa con la trayectoria profesional del empleado; en la menor importancia que se da a la transmisión y reproducción de una cultura organizacional de origen; en la menor lealtad e implicación personal exigidas al empleado. Por el contrario, el espacio empresarial japonés viene marcado por la exigencia y el control de casi cada aspecto de la vida laboral y social del empleado, todo ello asentado sobre una estricta concepción jerárquica de las relaciones sociales y de la lealtad debida. Esta rigidez entronca con una vinculación entre el movimiento migratorio del sujeto y su movilidad social en la empresa. La expatriación japonesa se ajusta estrictamente a una career-path migration (Salt, 1983).

Llama la atención el alcance del control que ejercen las empresas japonesas sobre los espacios de sociabilización, marcado incluso por numerosas prohibiciones. Esto contrasta también con el caso español, pues aun cuando la situación de inseguridad 
pública lo amerita, las empresas no sobrepasan el límite de la sugerencia de restringir los movimientos de sus empleados más allá del espacio y el tiempo laborales.

Pero si para el caso japonés vemos claramente cómo las relaciones de los expatriados con sus organizaciones tienen una injerencia restrictiva en sus dinámicas comunitarias, en la contraparte española observamos un influjo indirecto con resultados generalmente inversos. La mayor flexibilidad de la empresa o menor influencia sobre el proceso de adaptación de los expatriados españoles genera en estos una mayor incertidumbre. Una herramienta para compensar la ansiedad que genera esta incertidumbre es el acompañamiento de la familia del expatriado en su traslado. Tanta es la necesidad de este tipo de acompañamiento, que las empresas otorgan importantes complementos económicos a sus empleados con familia, cuando no directamente proveen de un empleo a las esposas que lo desean. Justo lo contrario sucede en el caso japonés: la prohibición de trabajar a las esposas. Esta prohibición es el reflejo, por un lado, de la reproducción de patrones culturales que marcan las relaciones de género y, por otra parte, de la importancia para la empresa de que ninguna institución (en este caso la familia) entre en competencia con la lealtad exigida a sus empleados. El resultado es un colectivo de expatriados, mayoritariamente varones y sin familia, entregados a la certidumbre que ofrecen unas directrices marcadas detalladamente por su empresa. La consecuencia, en términos de colectivismo, es un panorama de atomización (como hemos representado en la figura I).

En lo que se refiere a la solución de sociabilidad del grupo de expatriados españoles, consideramos que viene determinada por esa flexible reglamentación empresarial que conduce a la incertidumbre. Así, la búsqueda de apoyo para la obtención de soluciones pragmáticas a problemas cotidianos empuja al expatriado a relacionarse más allá del grupo laboral. Aquí el papel de la familia, y en particular el de la mujer, resulta clave para la construcción de redes para obtener información que facilite la adaptación al contexto local, pero también para construir un espacio trasnacional de matices comunitarios y con una tendencia a relaciones de solidaridad. Todo ello provee de la satisfacción de necesidades gregarias y afectivas, lo que nos acerca al concepto de comunidad que proponíamos al inicio de nuestro artículo. No obstante, parece que la pertenencia a un estatus tan sólidamente definido, unido a la fugacidad de la migración, impide profundizar o consolidar el sustrato migratorio. En este sentido, nos parece especialmente significativo el tabú referido a la ayuda económica al compatriota de similar estatus. En cuanto a la fugacidad de la migración (por la permanencia en el destino limitada en años), esta no es tanta ni tan rígida como la japonesa, por lo que algunos de los españoles expatriados que prolongan su estancia pueden actuar como importantes elementos de cohesión grupal. En suma, 
consideramos como hipótesis plausible el establecimiento de la correlación entre la fortaleza del respaldo empresarial (que implica un fuerte control sobre la acción social del empleado) y la débil búsqueda de espacios de relación con connacionales para la construcción de una comunidad con alto grado de cohesión grupal.

Otra conclusión emanada de nuestra comparación radica en la fortaleza de la sociabilidad, entendida como socialización lúdica (Simmel, 2003), como elemento que permite transitar del circuito trasnacional empresarial (motivación más pragmática) a la comunidad (motivación más íntima). Es importante remarcar que para que la sociabilidad sea tal, debe ser libre para el individuo, por lo que las formas de socialización extralaborales inducidas y controladas por la empresa japonesa no pueden ser asumidas, en sentido estricto, como formas de sociabilidad. Si bajo cierto concepto de comunidad el elemento de la vivencia subjetiva del integrante de dicha comunidad subyace como esencial (Doncel, 2013), también en nuestro estudio hemos encontrado que la búsqueda de satisfacción de necesidades afectivas, factor de cohesión grupal a decir de Carron, Brawley y Widmeyer (1998), se ha revelado como motor de sociabilidad de los expatriados españoles; y no en el caso de los japoneses, lo que conduce al expatriado de esta nacionalidad a la soledad e incluso a la depresión (Hirai, 2013).

Finalmente, podemos contrastar los espacios trasnacionales simbólicos que construye cada una de las comunidades de expatriados estudiadas. La migración empresarial japonesa, fuertemente condicionada por su cultura empresarial nacional, busca generar un espacio cultural japonés suprageográfico en torno al mundo laboral lo menos asimilado posible al contexto cultural local. Sin embargo, limitaciones en su arraigo histórico y en el tamaño de la comunidad en el Амм obstaculizan el despliegue de la propia cultura. De ahí que la vida social de los expatriados se encuentre tan empobrecida. En el caso español, es más difícil delinear con precisión ese espacio simbólico, pero sí se da en el expatriado ibérico un proceso, apoyado en la presencia familiar, de construcción de un espacio caracterizado por el equilibrio entre la necesidad de sentir una suerte de identificación colectiva entre connacionales y la apertura a la interacción con elementos ajenos al grupo principal de referencia. Esta apertura se manifiesta hacia la sociedad receptora, pero enmarcada siempre por el estatus y el estilo de vida identificado con una elite globalizada. 


\section{Referencias}

Álvarez, Marta y Sandalio Gómez (2008), El proceso de expatriación en empresas multinacionales: visión del expatriado, Ernst \& Young/Iese Business School <http://www.iese.edu/en/files/Informe\%2oExpatriados\%28para\%2oweb\%29_tcm4-38593.pdf> [consulta: 05/04/2017].

Beaverstock, Jonathan (20II), "Servicing British Expatriate'Talent' in Singapore: Exploring Ordinary Transnationalism and the Role of the 'Expatriate' Club", Journal of Ethnic and Migration Studies, 37(5), pp. 709-728. DoI: http://dx.doi.org/I0.1080/1369183X.2011.559714.

Beltrán, Joaquín y Amelia Sáiz (2009), “Introducción: apuntes teóricos y consideraciones metodológicas”, enः Joaquín Beltrán y Amelia Sáiz (eds.), Empresariado asiático en España, Barcelona, Fundació сіров, pp. 17-34. $<$ https://www.cidob.org/es/publicaciones/serie_de_publicacion/interrogar_la_actualidad/empresariado_asiatico_en_espana $>$ [consulta: 05/04/2017].

Bermúdez, Rosa (2010), "Migración calificada e integración en las sociedades destino", Sociedad y Economía, I9, pp. 135-I50.

Black, J. Stewart y Mark Mendenhall (1990),"Cross-Cultural training effectiveness: a review and a theoretical framework", Academy of Management Review, I5(I), pp. II3-136. DoI: http://dx.doi.org/10.2307/258109.

Bonache, Jaime y José Pla (2002), "La selección de directivos en las multinacionales desde la lógica de la teoría de la internacionalización”, Cuadernos de Economía y Dirección de la Empresa, 12, pp. 337-353.

Carron, Albert; Laurence Brawley y Neil Widmeyer (2008), "The measurement of cohesiveness in sport groups", en: Joan Duda (ed.), Advances in sport and exercise psychology measurement, Morgantown, Fitness Information Technology, pp. 213-226.

Chung, Peter (2014), "Living Globally: exploring the need for foreign enclaves in Shanghai", Columbia University Academic Commons <https://academiccommons.columbia.edu/catalog/ac:17529I > [consulta: 03/12/2017].

Doncel, Juan (2013), "Una escala de medición del grado de cohesión grupal de tres comunidades extranjeras en Área Metropolitana de Monterrey”, Trayectorias, I5(37), pp. 3-30.

Doncel, Juan (2015a), Once migraciones internacionales. Once comunidades extranjeras, México, Siglo xxi Editores. 
Doncel, Juan (2015b),"Cohesión grupal y distancia cultural. Factores determinantes en los procesos adaptativos a la sociedad de acogida de las comunidades de extranjeros", en: Juan Doncel (coord.), El mundo en Monterrey: migraciones y comunidades, México, Plaza y Valdés, pp. 173-199.

Faist, Thomas (2000), "Transnationalism in international migration: implications for the study of citizenship and culture", Ethnic and Racial Studies, 23(2), pp. 189-222. DoI: http://dx.doi.org/I0.1080/or4198700329024. Findlay, Allan y Sophie Cranston (2015), "What's in a research agenda?: An evaluation of research developments in the arena of skilled international migration", International Development Planning Review, 37(I), pp. I7-3I. DOI: http://dx.doi.org/Io.3828/idpr.20I5.3.

Findlay, Allan y W. T. S. Gould (1989), "Skilled International Migration: A Research Agenda", Area, 2I(I), pp. 3-II.

Glick Schiller, Nina, Linda Basch y Cristina Blanc-Szanton (1992), “Transnationalism: A New Framework for Understanding Migration”, Annals of the New York Academy of Sciences, 645, pp. I-24. DoI: http://dx.doi. org/I0.IIII/j.I749-6632.1992.tb33484.x.

Harris, Hilary y Len Holden (200I), "Between Autonomy and Control: Expatriate Managers and Strategic IHRM in SMes", Thunderbird International Business Review, 43(I), pp. 77-IOO DoI: http://dx.doi.org/I0.1002/15206874(200IOI/O2) 43:1<77:ःAID-TIE6>3.0.CO;2-G.

Hayton, James, Gerard George y Shaker Zahra (2002), "National culture and entrepreneurship: A review of behavioral research", Entrepreneurship Theory and Practice, 26(4), pp. 33-52. DoI: http://dx.doi. org/10.1177/104225870202600403.

Heller, Ágnes (2002), Sociología de la vida cotidiana, Barcelona, Península.

Hirai, Shinji (2013), "Migración y corporaciones japonesas en el noreste de México: las prácticas sociales y culturales de los migrantes y su salud mental", en: Lucía Chen y Alberto Saladino (comps.), La nueva Nao: de Formosa a América Latina, Taipei, Universidad de Tamkang, pp. 7I-98. Hirai, Shinji (2015), "La vida migratoria de kagai chuzai in: Migración, empresas y comunidad japonesas en Nuevo León”, en: Juan Doncel (coord.), El mundo en Monterrey: migraciones y comunidades, México, Plaza y Valdés, pp. 97-I2I.

Ho, Christina (2006),"Migration as Feminisation? Chinese Women's Experiences of Work and Family in Australia", Journal of Ethnic and Migration Studies, 32(3), pp. 497-514. DoI: http://dx.doi.org/I0.1080/13691830600555053. 
Hofstede, Geert (1980), Culture's consequences: International differences in work-related values, Beverly Hills, Sage.

McSweeney, Brendan (2002), "Hofstede's model of national cultural differences and their consequences: A triumph of fatith - A failure of analysis", Human Relations, 55(I), pp. 89-II8. Dor: http://dx.doi. org/IO.II77/oor8726702551004.

Mead, Richard (2009), International management: cross-cultural dimensions, Chichester, Wiley and Sons.

Mendoza, Cristóbal (2018), "Migración y movilidad de los trabajadores cualificados extranjeros de las empresas en México", Iztapalapa, 84, pp. 15-47.

Mendoza, Cristóbal, Barbara Staniscia y Anna Ortiz (2016),"Migración y movilidad de las personas calificadas: nuevos enfoques teóricos, territorios y actores", Biblio 3 W. Revista Bibliográfica de Geografía y Ciencias Sociales, 2I(II66), pp. I-22.

Mirabal, Alberto y Gerardo Zapata (2009), "Estructura y Expatriación: Riesgos en la asignación externa para expatriados que aprenden", Ciencias Económicas, 27(2), pp. 21-32.

огм (2010), "Migration and transnationalism: opportunities and challenges" (Intersessional workshop Background paper), Organización Internacional para las Migraciones. < https://www.iom.int/jahia/webdav/shared/ shared/mainsite/microsites/IDM/workshops/migration_and_transna tionalism_030910/background_paper_en.pdf $>$ [consulta: 03/12/2017].

Ortiz Guitart, Anna y Cristóbal Mendoza (2007), "Mujeres expatriadas en México: trabajo, hogar y vida cotidiana”, Migraciones internacionales, 4(2), pp. 5-32. DoI: http://dx.doi.org/IO.17428/rmi.v4ir3.II65.

Peixoto, João (200I), “The International Mobility of Highly Skilled Workers in Transnational Corporations: The Macro and Micro Factors of the Organizational Migration of Cadres", International Migration Review, 35(4), pp. I030-I053. DoI: http://dx.doi.org/IO.IIII/j.I747-7379.200I. tbooos I.x.

Perlmutter, Howard V. (1969), "The Torturous Evolution of the Multinational Coroporation", Columbia Journal of World Business, 4(I), pp. 9-18.

Portes, Alejandro, Luis Eduardo Guarnizo y William J. Haller (2002), "Transnational Entrepreneurs: An Alternative Form of Immigrant Economic Adaptation", American Sociological Review, 67(2), pp. 278-298. DoI: http://dx.doi.org/I0.2307/3088896. 
Rodríguez, Ernesto y Salvador Cobo (2012), Extranjeros residentes en México. Una aproximación cuantitativa con base en los registros administrativos del INM. México, Centro de Estudios Migratorios, INM.

Salt, John (1983), "High level manpower movements in northwest Europe and the role of careers", International Migration Review, I7(4), pp. 633-636. DoI: http://dx.doi.org/10.2307/2545822.

Simmel, Georg (2003), Cuestiones fundamentales de sociología, Barcelona, Gedisa. Williams, Angela Marsha (2004),"The French Expatriate Assignment: Helping Accompanying Spouses to Adapt by Assuming the Role of Anthropologist", Brigham Young University - All Theses and Dissertations 213. $<$ http://scholarsarchive.byu.edu/etd/213>.

Willis, Katie y Brenda Yeoh (2002), "Gendering Transnational Communities: A Comparison of Singaporean and British Migrants in China", Geoforum, 33(4), pp. 553-565. Dor: http://dx.doi.org/Io.IoI6/Soor67185(02)00039-8.

JUAN SORdo

Doctor en Estudios Humanísticos (especialidad en estudios de Ciencia y Cultura) por el Tecnológico de Monterrey. Miembro del Centro de Estudios Interculturales del Noreste en la Universidad Regiomontana e investigador posdoctoral del proyecto "Estudiantes indígenas en Educación Media Superior y Superior en Nuevo León. Panorama de inserción socioeducativa y construcción de identidad étnica”, financiado por Conacyt. Fue becario del Programa Nacional de Posgrados de Calidad y colaborador de la Cátedra de Ciencia y Cultura del Tecnológico de Monterrey. Sus publicaciones más recientes son "Adquisición escolar del español y minusvaloración de las propias competencias lingüísticas en estudiantes indígenas emigrados a Nuevo León" (en Revista Mexicana de Investigación Educativa); "Estudiar para ser alguien. Patrones discursivos sobre la propia etnicidad y la movilidad social en indígenas estudiantes de niveles superiores migrantes a Monterrey" (en Fragmento de la comunicación rupestre); "Cultura y política en el proyecto filosófico de Peter Sloterdijk" (en Desacatos).

Juan Antonio Doncel de la Colina

Doctor en Antropología Social por la Universidad de A Coruña (España). Es profesor, investigador y director del Centro de Estudios Interculturales del Noreste en 
la Universidad Regiomontana, así como miembro del sNi, nivel 2. En la actualidad dirige un proyecto de Ciencia Básica (Conacyt): "Estudiantes indígenas de Educación Media Superior y Superior en Nuevo León. Panorama de inserción socioeducativa y construcción de identidad étnica". Ha publicado cinco libros (tres como único autor y dos como coautor), además de una veintena de artículos en revistas científicas y capítulos de libro. Sus publicaciones más recientes son "Percepción y consumo de telenovelas e identidad étnica de universitarios y universitarias indígenas: el caso de Un refugio para el amor" (en Comunicación y Sociedad); "El rap indígena: activismo artístico para la reivindicación del origen étnico en un contexto urbano" (en Andamios); "Significaciones de mass media en preparatorianos y universitarios indígenas emigrados a Monterrey" (en Estudios Fronterizos).

Citar como: Sordo, Juan y Juan Antonio Doncel de la Colina (2018),"Expatriados empresariales y comunidades extranjeras en Monterrey: los casos español y japonés", Iztapalapa. Revista de Ciencias Sociales y Humanidades, núm. 85, año 39, julio-diciembre de 2018, IsSN: 2007-9176; pp. I39-168. Disponible en $<$ http://revistaiztapalapa.izt.uam.mx/index.php/izt/issue/ archive $>$. 